Review

\title{
Ultrahigh Resolution Fiber-Optic Quasi-Static Strain Sensors for Geophysical Research
}

\author{
Zuyuan $\mathrm{HE}^{1 *}$, Qingwen $\mathrm{LIU}^{1}$, and Tomochika TOKUNAGA ${ }^{2}$ \\ ${ }^{1}$ State Key Lab of Advanced Optical Communication Systems and Networks, Shanghai Jiao Tong University, Shanghai, \\ 200240, China \\ ${ }^{2}$ Department of Environment Systems, University of Tokyo, Chiba 277-8563, Japan \\ *Corresponding author: Zuyuan HE_ E-mail: zuyuanhe@sjtu.edu.cn
}

\begin{abstract}
A review of our recent work on ultrahigh resolution optical fiber sensors in the quasi-static region is presented, and their applications in crustal deformation measurement are introduced. Geophysical research such as studies on earthquake and volcano requires monitoring the earth's crustal deformation continuously with a strain resolution on the order of nano-strains (ne) in static to low frequency region. Optical fiber sensors are very attractive due to their unique advantages such as low cost, small size, and easy deployment. However, the resolution of conventional optical fiber strain sensors is far from satisfactory in the quasi-static domain. In this paper, several types of recently developed fiber-optic sensors with ultrahigh resolution in the quasi-static domain are introduced, including a fiber Bragg grating (FBG) sensor interrogated with a narrow linewidth tunable laser, an FBG based fiber Fabry-Perot interferometer (FFPI) sensor by using a phase modulation technique, and an FFPI sensor with a sideband interrogation technique. Quantificational analyses and field experimental results demonstrated that the FBG sensor can provide nano-order strain resolution. The sub-nano strain resolution was also achieved by the FFPI sensors in laboratory. Above achievements provide the basis to develop powerful fiber-optic tools for geophysical research on crustal deformation monitoring.
\end{abstract}

Keywords: Ultrahigh resolution, fiber Bragg grating, frequency modulation, crustal deformation measurement

Citation: Zuyuan HE, Qingwen LIU, and Tomochika TOKUNAGA, "Ultrahigh Resolution Fiber-Optic Quasi-Static Strain Sensors for Geophysical Research,” Photonic Sensors, DOI: 10.1007/s13320-013-0132-5.

\section{Introduction}

In geophysical research, the measurement of crustal deformation is of great relevance to studies of earthquakes and volcanoes [1,2]. For those applications, strain sensors with a resolution on the order of nano-strain $(n \varepsilon)$ in a static to low-frequency region are required. Conventional sensors for this purpose include extensometers and interferometers installed underground. These sensors, however, are large in size, ranging from several tens to hundreds of meters in length, and thus difficult to be installed widely, especially in the deep underground. Moreover, because such a sensor can only give integrated strain information over its length, no spatially resolved deformation information can be obtained. On the other hand, fiber-optic sensors, for example, fiber Bragg grating (FBG) strain sensors, have well-known advantages such as small size, low cost, easy installation, high stability, and good

Received: 18 July 2013 / Revised version: 31 August 2013

(C) The Author(s) 2013. This article is published with open access at Springerlink.com 
linearity over a large strain range. They have already been widely adopted in applications like smart materials and structural healthy monitoring [3]. Naturally, they are also very attractive for geophysical applications if they can provide the required strain resolution.

However, the performance of the conventional fiber sensor is not so satisfactory for quasi-static strain measurement, even though FBG sensors have realized even better than pico-strain $(p \varepsilon)$ resolution for dynamic strain sensing [4-6]. A dynamic strain signal, i.e., vibration, repeats itself periodically, so it is self-referenced. In contrast, a quasi-static strain signal exhibits just as a random arbitrary signal in a given observation period, and the environmental disturbance such as temperature variation is also in the quasi-static region. Therefore, an extra reference is required for quasi-static strain sensing, making the sensor more challenging than a dynamic sensor. A reference of the spectroscopic frequency associated with an atomic or molecular transition has been a popular reference for static strain sensing [7-9], but this type of reference cannot compensate sensor's output drift due to environmental interference, and the narrow bandwidth of the transition also limits the dynamic range of the sensor.

In this paper, we introduce our recent research on quasi-static strain sensors for crustal deformation measurement, including sensors based on the FBG and fiber Fabry-Perot interferometer (FFPI), respectively. For each type of the sensor, a strain-free sensor head is employed working as the reference to compensate the long-term drift of both laser source and the sensor heads. A large dynamic range is guaranteed by the linear strain-wavelength response of sensor heads and the interrogation methods. With the FBG sensor, the crustal strain caused by the ocean tide was clearly observed with a static strain resolution better than $10 \mathrm{n} \varepsilon$ at Aburatsubo Bay, Japan, and the strain resolution up to sub-nano-strain was achieved with the FFPI sensor in laboratory. Those work showed that the optical fiber sensors have great potential for the applications of geophysical research.

\section{FBG-based ultrahigh resolution strain sensor}

\subsection{Principle and configuration of FBG sensor}

FBGs are fabricated by creating periodic variations in the refractive index of the core of an optical fiber [10]. Figure 1(a) shows the internal structure of an optical fiber with a grating written inside. When the lightwave propagates through the grating, at a particular wavelength named the Bragg wavelength $\lambda_{B}$, reflected lightwaves are in phase and enhanced. For a uniform grating, the reflection spectrum is shown in Fig. 1(b).

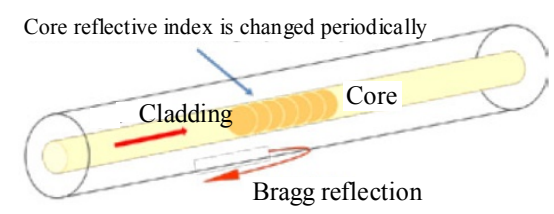

(a)

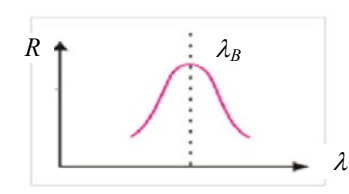

(b)

Fig. 1 Principle of FBG sensors: (a) the structure of the FBG, and (b) the spectrum of the uniform FBG.

For an FBG, the Bragg wavelength $\lambda_{B}$ is given as

$$
\lambda_{B}=2 n_{\text {eff }} \Lambda
$$

where $n_{\text {eff }}$ is the effective index of refraction of the FBG, and $\Lambda$ is the grating period.

When the FBG is strained or experiences a temperature change, its $\lambda_{B}$ will shift accordingly. The relative change in the Bragg wavelength is expressed as [11]

$$
\Delta \lambda_{B} / \lambda_{B}=(\alpha+\xi) \Delta T+\left(1-p_{\varepsilon}\right) \varepsilon
$$

where $\Delta T$ is the temperature change, $\varepsilon$ is the longitudinal strain, $\alpha$ is the thermal expansion coefficient, $\xi$ is the thermo-optic coefficient, and $p_{\varepsilon}$ is the effective elasto-optic constant of the fiber material, respectively. Equation (2) shows that the 
Bragg wavelength is sensitive to both strain and temperature linearly. For static strain measurement, it is necessary to distinguish the shift caused by strain from that by temperature. A simple but efficient solution to this problem is to add a strain-free reference FBG for reference.

As shown in Fig. 2, a pair of identical FBGs are employed in the sensing system; one is for strain sensing, and the other is strain-free working as a reference [12]. The two FBGs are mounted closely to each other to let them feel the same temperature variation, so the differential Bragg wavelength change indicates the strain information. A narrow linewidth tunable laser is used to interrogate the FBGs, and the lightwave reflected from the FBG is detected by a photo-detector. While the wavelength of the tunable laser sweeps over the principal peaks of the FBGs' reflective spectra, their reflectivity is sampled at a discrete sequence of the wavelength $\lambda_{i}$ with a step of $d \lambda$. The reflectivity of the sensing FBG is labeled as $R\left(\lambda_{i}\right)$, while the reflectivity of the reference FBG is labeled as $R_{R}\left(\lambda_{i}\right)$.

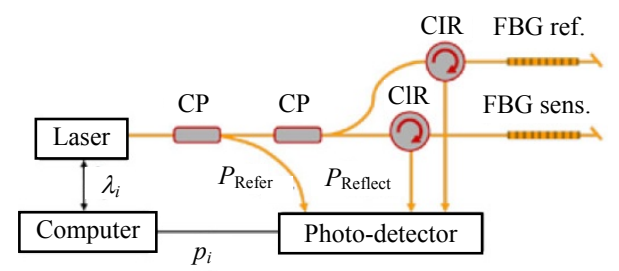

Fig. 2 Configuration of the narrow linewidth tunable laser based FBG static strain sensor: CP, coupler; CIR, circulator.

\subsection{Cross-correlation algorithm}

The cross-correlation algorithm is used to demodulate the differential wavelength shift $\Delta \lambda$ from the measured spectra between the reference FBG and the sensing FBG, because this algorithm is proved to have very good resolution compared with other algorithms such as the centroid detection algorithm and the least square fitting algorithm [12]. Once the spectrum of the reference FBG and that of the sensing FBG are recorded, the cross-correlation product is calculated to determine the Bragg wavelength difference between the two spectra:

$$
C(j)=\sum_{i=-N}^{N} R\left(\lambda_{i+j}\right) R_{R}\left(\lambda_{i+\varepsilon}\right) .
$$

In (3), it is assumed that both $R\left(\lambda_{i}\right)$ and $R_{R}\left(\lambda_{i}\right)$ are equal to zero if the indices lie outside their ranges. This assumption is acceptable as long as the sampling range covers the whole principal peaks of both FBGs. $C(j)$ has the maximum at $j=\varepsilon$, where the two spectra overlap completely, and $\varepsilon$ is demodulated from the index when $C(j)$ is at its maximum. Due to the random errors in the measured reflectivity, the retrieved $\varepsilon$ deviates from the actual strain, and the deviation range is the resolution of the sensor. It should be mentioned that although the index of the maximum $C(j)$ falls into an integer which is the nearest to $\varepsilon, \varepsilon$ can be precisely calculated either by interpolation or by curve-fitting around the maximum.

\subsection{Analysis on strain resolution}

The measured reflectivity of the FBG suffers from the intensity noise of the laser and the photo detector, and from the wavelength accuracy of the tunable laser. These are the main origins of the strain resolution deterioration.

The intensity noise in the measurement of the optical power includes the shot noise of the light, the noise of the photo-detector, and the quantization noise in the analog to digital (A/D) convert process.

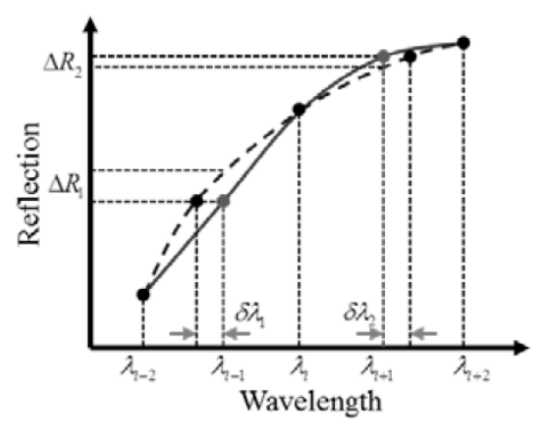

Fig. 3 Wavelength repeatability induced reflectivity error in measurement (broken line and solid line: two measured spectra with wavelength fluctuation, where $\Delta R_{1}$ and $\Delta R_{2}$ are induced by $\delta \lambda_{1}$ and $\delta \lambda_{2}$, respectively).

The wavelength accuracy is mainly affected by 
the laser source's phase noise, which is negligible when a narrow linewidth laser source is used, and the wavelength drift. For a tunable laser, the wavelength drift exhibits as wavelength repeatability, which is the random uncertainty of the measured wavelength in repeating sweeps. The uncertainty of

$$
\lambda_{R}=\sqrt{d \lambda} \cdot \sqrt{\frac{\sqrt{n^{2}+0.26 n+0.3}}{(0.972 n+0.28)^{2}}} \cdot \lambda_{\text {width }} \cdot \sigma^{2}\left(\Delta R_{\text {elec }}\right)+\frac{20 n^{2}-9}{(1.944 n+0.56)^{2}} \cdot \frac{\sigma^{2}(\delta \lambda)}{\lambda_{\text {width }}}
$$

where $\lambda_{R}$ is the wavelength resolution of the sensor, $d \lambda$ is the wavelength sweep step of the laser, $\sigma(\Delta R)$ is the standard deviation of the relative intensity noise, and $\sigma(\delta \lambda)$ is the standard deviation of wavelength accuracy. From the wavelength resolution, the strain resolution can be simply deduced by the strain-wavelength coefficient of the FBG, which is about $1.2 \mathrm{pm} / \mu \varepsilon$.

Based on (4), we obtain the guidelines for the sensor design and optimization.

\section{A. FBG spectrum}

An FBG with a smoother spectrum is preferable to achieve higher resolution, and the optimized bandwidth is determined by the ratio of intensity noise level and frequency noise level. An FBG with a narrower bandwidth is preferred to achieve higher resolution when using a laser source with good wavelength repeatability, while a broader bandwidth FBG is suitable to make full use of the low intensity noise photo-detectors.

\section{B. Sweep step of the laser source}

The sweep step $d \lambda$ plays an important role in the resolution. A smaller sweep step $d \lambda$ while keeping the integration time of the photo-detector constant produces better resolution. It consumes, however, longer time to complete one sweep.

\subsection{Field experiment}

The FBG sensor was adopted in field experiments for crustal deformation measurement after laboratory demonstration. The field experiments were carried out at the Aburatsubo Crustal Deformation Observatory, Earthquake Research Institute, University of Tokyo. The the measured wavelength can be converted into an error in reflectivity, and the converting coefficient is the differential of the FBG's spectrum, as shown in Fig. 3.

The resolution of the FBG sensor in Fig. 2 is deduced to be [12]

observatory locates at the tip of the Miura Peninsula, about $60 \mathrm{~km}$ southwest to Tokyo, Japan. As shown in Fig. 4, one vault with several sections is built on the coast at Aburatsubo Bay. Extensometers with the length of $38 \mathrm{~m}$ are set in one of the sections for crustal deformation measurement. The FBG strain sensor is installed in another section. When the ocean tide level varies, the pressure applied to the sea bottom changes accordingly, resulting in the deformation of the rock mass. This phenomenon provides a good reference load to verify the performance of the sensors [13].

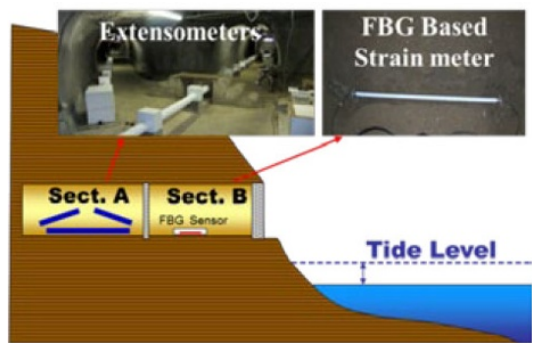

Fig. 4 Experiment configuration for measurement of crustal deformation at Aburatsubo Bay, Japan

In the first experiment, the configuration of the system is similar to that shown in Fig. 2. The maximum reflection of the FBGs is about $70 \%$ with a bandwidth of $0.23 \mathrm{~nm}$.

One FBG is mounted between two piers that are anchored 30-cm deep to the rock, and the other FBG is strain-free for reference. A narrow-linewidth tunable laser (Agilent $81680 \mathrm{~A}$, linewidth $100 \mathrm{kHz}$ ) is used to interrogate the FBGs. The reflected light beams from both FBGs are detected by the photo-detectors (Agilent 81635A). About 10\% of the power from the laser source is used as intensity reference for power ratio detection to eliminate 
effects of power fluctuation during wavelength sweeping. The tunable laser sweeps with a step of $0.1 \mathrm{pm}$ at a speed of $500 \mathrm{pm} / \mathrm{s}$. Both the wavelength and the corresponding reflected power are logged at each step. The whole process is controlled by a LabVIEW program running on a laptop computer.

Figure 5(a) illustrates part of the measured strain by FBG sensors during the experiment. For comparison, Fig. 5(b) shows the measured strain by the extensometer during the same period. The two curves have similar shapes as expected, indicating that the deformation induced by the ocean tide is clearly observed by our FBG strain sensor. The small difference in amplitude is possibly caused by the difference in mounting methods, in the baseline length, or in the location. The strain resolution of the FBG sensor is calculated to be better than $10 \mathrm{n} \varepsilon$. This is the first time that $10 \mathrm{n} \varepsilon$ order static strain resolution is demonstrated in practice with FBG sensors [14].

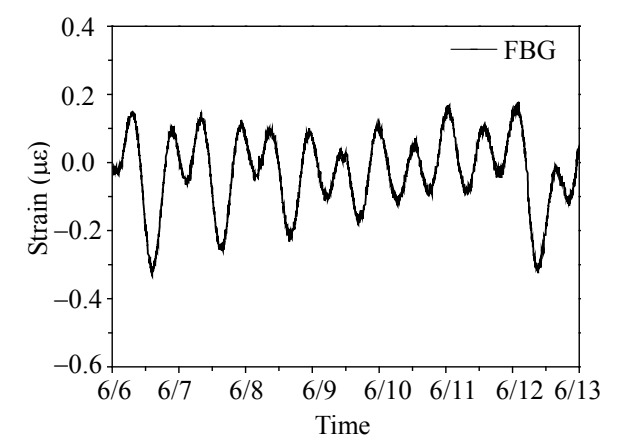

(a)

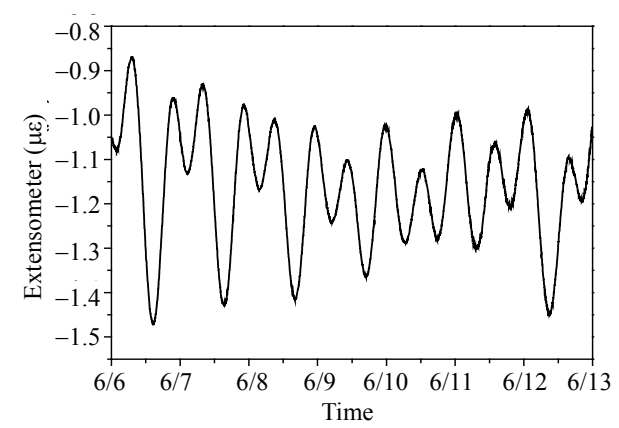

(b)

Fig. 5 In-situ experimental results with the FBG sensor: (a) measured strain by the FBG sensor during June 6th to June 13th, 2011, and (b) measured strain by an extensometer in the same period.

\section{FFPI sensors interrogated with a frequency modulation technique}

According to the analysis on the resolution of FBG sensors, the performance is mainly limited by the laser's wavelength repeatability during the sweeping. The bandwidth of a practical FBG ranges from tens to hundreds of $\mathrm{pm}$, and the tunable laser has to cover the spectra of two FBGs, one for strain sensing and the other for reference. In the FBG sensor, a mechanically-tuned external cavity diode laser is used as the light source. It is hard to further improve its wavelength repeatability. To obtain even higher resolution, we have to reduce the required tunable range and thus mitigate the requirements of the wavelength repeatability. To do so, we developed sensors based on fiber Fabry-Perot interferometers (FFPI) which have narrower bandwidth.

\subsection{Interrogation of FFPI with frequency modulation technique}

The fiber Fabry-Perot interferometer is formed by placing two FBGs in one fiber. It has the same strain/temperature sensitivity as the FBG, while the main difference is that the FFPI has a series of resonances, and the bandwidth of the resonance can be on the order of $\mathrm{MHz}$, much narrower than that of the FBG. Since the bandwidth of the FFPI falls into the region of radio frequency, the FFPI can be interrogated by a more efficient method using frequency modulation (FM) technique.

The principle of the FM interrogation technique is shown in Fig. 6. The light frequency of the laser is modulated with the frequency of $\Omega$, and the reflected power from the FFPI is monitored. If the laser frequency deviates from the resonance frequency of the Fabry-Perot cavity, the reflected power consists of the $\Omega$ component as well as the $2 \Omega$ component. The phase and the amplitude of the $\Omega$ component show how the laser frequency deviates from the resonance frequency. Otherwise, if the central frequency of laser lies exactly at the resonance of 
the Fabry-Perot cavity, the reflected optical power is modulated with the frequency of $2 \Omega$ and does not consist of the $\Omega$ component.

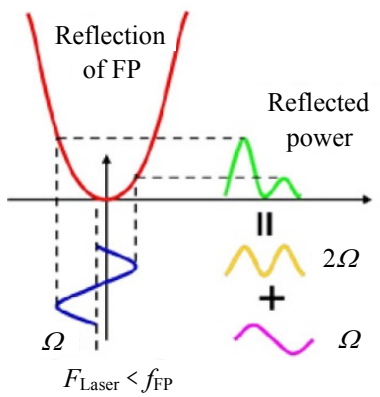

Fig. 6 Principle of frequency modulation technique for the interrogation of FFPI.

\subsection{Sensor configuration and experimental results}

The schematic configuration of the proposed FFPI sensor is shown in Fig. 7. It consists of a pair of identical FFPIs; one is for the sensing of strain, and the other is strain-free working as a reference and a temperature sensor for temperature compensation. Each FFPI, fabricated by Fujikura Ltd., is formed by writing two identical FBGs $20-\mathrm{cm}$ distant in a single mode fiber. The parameters of the FBGs are: nominal central wavelength $1549.85 \pm$ $0.04 \mathrm{~nm}$, bandwidth $0.25 \mathrm{~nm}$, and peak reflectivity 99.5\%. A narrow linewidth tunable fiber laser is used to interrogate the FFPIs with the FM interrogation technique. The lightwave from the laser source is phase-modulated with a phase modulator (PM) driven by a sinusoidal function generator (FG), and thus two sidebands are generated besides the carrier. When these lightwaves are reflected by an FFPI, an intensity modulation appears in the reflected light. By demodulation of this intensity-modulated light, an output signal is obtained, which is a function of the frequency deviation between the laser and the resonance of FFPI [15].

In the validation experiments, both FFPIs are strain-free packaged to ascertain the performance of the sensing system. A fiber laser (NKT, E15, linewidth $1 \mathrm{kHz}$ ) with an integrated piezo tuner is used to interrogate the two FFPIs simultaneously via a 3-dB coupler. A function generator (Agilent, $33250 \mathrm{~A}$ ) is used to drive the $\mathrm{PM}$ with a $2-\mathrm{MHz}$ sinusoidal waveform. The lightwaves reflected by FFPIs are detected by high-speed photo diodes (New Focus, 1181) via circulators.

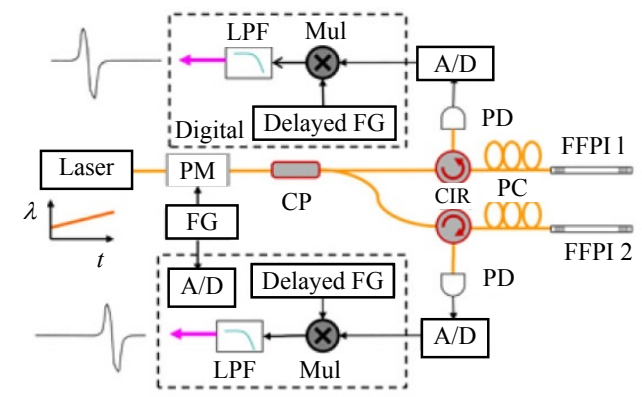

Fig. 7 Configuration of sensor: CP, coupler; PM, phase modulator; CIR, circulator; PD, photo diode; FG, function generator; $\mathrm{PC}$, polarization controller; $\mathrm{A} / \mathrm{D}$, analog to digital convertor; Mul, multiplier; LPF, low pass filter.

The evolution of the resonance difference between the two FFPIs against time is monitored and logged 2 times per minute. The program is designed to automatically select the first complete resonance peak from the demodulated signals for each FFPI, and then calculate the resonance difference using the cross-correlation algorithm.

This sensing system has a large dynamic range of hundreds of $\mu \varepsilon$, as long as the high reflection regions of all the FBGs remain overlapped under applied strain. The standard deviation of the measured resonance difference over 2 hours between

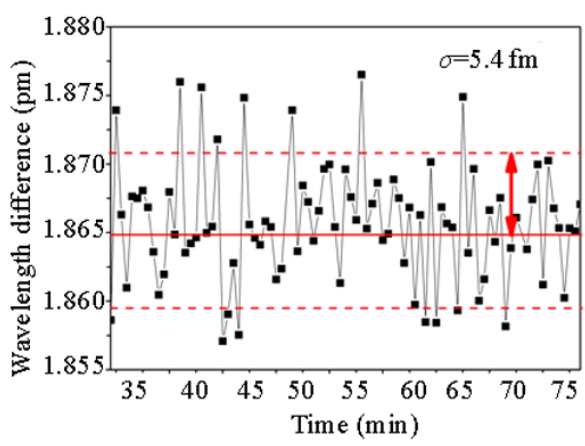

Fig. 8 Part of the extracted resonance difference between the FFPIs (the standard deviation after temperature compensation over 2 hours is $5.4 \mathrm{fm}$ ). 
the two FFPIs is only $5.4 \mathrm{fm}$ after temperature compensation, as shown in Fig. 8. The FFPI's strain sensitivity is measured to be $0.93 \pm 0.02 \mathrm{pm} / \mu \varepsilon$, so a corresponding strain resolution of $5.8 \mathrm{n} \varepsilon$ is achievable with the system, if the sensor head is designed properly.

\section{FFPI sensor with sideband interrogation technique}

With the FFPI as sensor head and frequency modulation interrogation method, the tuning range of laser can be as small as the free spectral range (FSR) of the FFPI. We newly proposed a sideband interrogation method, with which the tuning range can be further reduced to the bandwidth of the FFPI.

\subsection{Simultaneously interrogation with sideband and carrier}

In the typical configuration for FFPI with the frequency modulation method, a phase modulator driven by sinusoidal waveform is employed to generate frequency modulation. During the phase modulation, sidebands are generated as well as the carrier, as shown in Fig. 9 (labeled PM). If an intensity modulator is driven by the exactly same form as the PM signal, two group of sidebands will appear, and each group of sidebands has the same form as that of the lightwave after phase modulation, but the central frequency is shifted by $\Omega_{S}$, as the intensity modulator (IM) labeled in Fig. 9. The sideband can also be used for interrogating the FFPI [16].

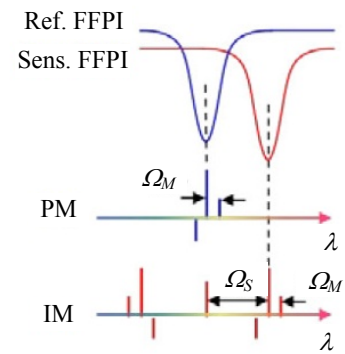

Fig. 9 Simultaneous interrogation of FFPIs with sideband and carrier.

\subsection{Sensor configuration and experimental result}

The sensor configuration with the sideband interrogation method is shown in Fig. 10. The lightwave from a narrow linewidth laser source is spilt into two paths to interrogate the two FFPIs, respectively. The reference FFPI is interrogated with one path of lightwave by using the typical frequency modulation technique. The sensing FFPI is interrogated by the other path of light modulated with an IM driven by an arbitrary waveform generator (AWG), which produces a phase modulated radio frequency signal.

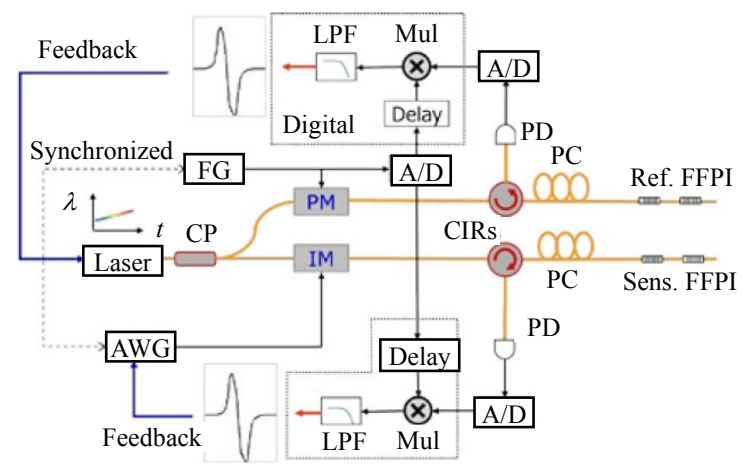

Fig. 10 System configuration: CP, coupler; PM, phase modulator; IM, intensity modulator; CIR, circulator; PC, polarization controller; $\mathrm{PD}$, photo-detector; FG, function generator; AWG, arbitrary waveform generator; Mul, multiplier; LPF, low pass filter.

After the intensity modulation, two groups of sidebands are generated together with the carrier. One group of the sidebands is used to interrogate the sensing FFPI, which has a resonance frequency different from the carrier frequency. The reflected lightwave from both FFPIs are demodulated with the same demodulation method.

The frequency of the narrow linewidth laser source is tuned to sweep around the resonance of the reference FFPI, as the PM lightwave shown in Fig. 9. Then, the central frequency of the sideband is adjusted to approximate the resonance frequency difference between the two FFPIs. As a result, one group of the sideband is shifted to sweep around the resonance of the sensing FFPI simultaneously, as the 
IM light shown in Fig. 9. The other group of the sideband has no contribution to the demodulated signal as long as they are far away from any resonance frequency of the sensing FFPI. During the laser wavelength sweeping, the demodulated signals from the two FFPIs are similar in shape but staggered in the horizontal position. A cross-correlation algorithm is employed to calculate the frequency difference of the demodulated signals. The extracted frequency difference plus $\Omega_{S}$ is the actual resonance difference between the two FFPIs.

The extracted frequency difference from the demodulated signals is shown in Fig. 11. The fluctuation range (standard deviation) is $29 \mathrm{kHz}$, which also determines the frequency resolution of the sensor system because the inaccuracy in the measurement of $\Omega_{S}$ is negligible. The strain sensitivity of the FFPIs is tested to be $116 \mathrm{MHz} / \mu \varepsilon$; thus, a corresponding strain resolution of $0.3 n \varepsilon$ is achievable with the sensor system, if the sensor head is designed properly.

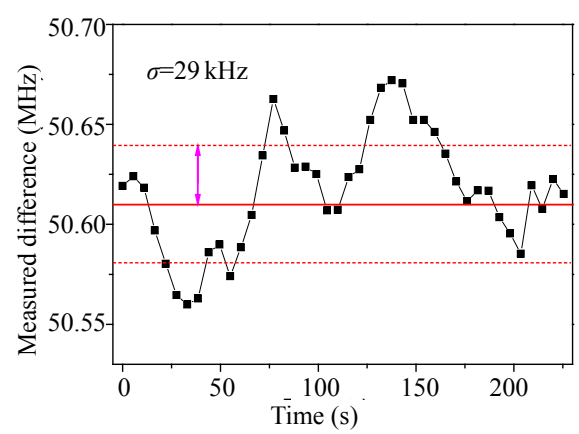

Fig. 11 Extracted frequency difference between demodulated signals.

\section{Summary}

In this review paper, our recent work on the ultrahigh resolution optical fiber strain sensor in the quasi-static region is introduced. The resolution of FBG sensor interrogated by narrow linewidth laser is theoretically analyzed, and the guidelines for the sensor design are discussed. With the FBG sensor, crustal deformation is measured with resolution about $10 \mathrm{n} \varepsilon$, which is the first time that $10 \mathrm{n} \varepsilon$ order static strain resolution is demonstrated in practice with FBG sensors. With the FFPI sensor interrogated by a sideband method, sub-nano order strain resolution is obtained in laboratory. Together with the common advantages of fiber sensors, our work show that optical fiber sensor has great potential for applications of geophysical research.

\section{Acknowledgment}

The authors thank Prof. Kazuo Hotate at the University of Tokyo, Japan, for the helpful discussions and comments. This work was supported by the Strategic International Cooperation Program from Japan Science and Technology Agency (JST), the Grant-in-Aid for Scientific Research (A) from Japan Science Promotion Society (JSPS), and the Global Center of Excellence (G-COE) Program from the Ministry of Education, Culture, Sports, Science and Technology (MEXT), Japan.

Open Access This article is distributed under the terms of the Creative Commons Attribution License which permits any use, distribution, and reproduction in any medium, provided the original author(s) and source are credited.

\section{References}

[1] R. Bilham, R. Bendick, K. Larson, P. Mohr, J. Braun, S. Tesfaye, et al., "Secular and tidal strain across the main Ethiopian rift," Geophysical Research Letters, vol. 26, no. 18, pp. 2789-2792, 1999.

[2] P. Ferraro and G. De Natale, "On the possible use of optical fiber Bragg gratings as strain sensors for geodynamical monitoring," Optics and Lasers in Engineering, vol. 37, no. 2-3, pp. 115-130, 2002.

[3] M. Majumder, T. K. Gangopadhyay, A. K. Chakraborty, K. Dasgupta, and D. K. Bhattacharya, "Fibre Bragg gratings in structural health monitoring - present status and applications," Sensors and Actuators A: Physical, vol. 147, no. 1, pp. 150-164, 2008.

[4] B. Lissak, A. Arie, and M. Tur, "Highly sensitive dynamic strain measurements by locking lasers to fiber Bragg gratings," Optics Letters, vol. 23, no. 24, pp. 1930-1932, 1998. 
[5] D. Gatti, G. Galzerano, D. Janner, S. Longhi, and P. Laporta, "Fiber strain sensor based on a pi-phase-shifted Bragg grating and the Pound-Drever-Hall technique," Optics Express, vol. 16, no. 3, pp. 1945-1950, 2008.

[6] I. C. Littler, M. B. Gray, J. H. Chow, D. A. Shaddock, and D. E. McClelland, "Pico-strain multiplexed fiber optic sensor array operating down to infra-sonic frequencies," Optics Express, vol. 17, no. 13, pp. 11077-11087, 2009.

[7] A. Arie, B. Lissak, and M. Tur, "Static fiber-Bragg grating strain sensing using frequency-locked lasers," Journal of Lightwave Technology, vol. 17, no. 10, pp. 1849-1855, 1999.

[8] G. Gagliardi, M. Salza, P. Ferraro, and P. De Natale, "Fiber Bragg-grating strain sensor interrogation using laser radio-frequency modulation," Optics Express, vol. 13, no. 7, pp. 2377-2384, 2005.

[9] T. T. Y. Lam, J. H. Chow, D. A. Shaddock, I. Littler, G. Gagliardi, M. B. Gray, et al., "High-resolution absolute frequency referenced fiber optic sensor for quasi-static strain sensing," Applied Optics, vol. 49, no. 21, pp. 4029-4033, 2010.

[10] G. Meltz, W. W. Morey, and W. H. Glenn, "Formation of Bragg gratings in optical fibers by a transverse holographic method," Optics Letters, vol. 14, no. 15, pp. 823-825, 1989.
[11] A. D. Kersey, M. A. Davis, H. J. Patrick, M. LeBlanc, K. P. Koo, C. J. Askins, et al., "Fiber grating sensors," Journal of Lightwave Technology, vol. 15, no. 8, pp.1442-1463, 1997.

[12] Q. Liu, T. Tokunaga, and Z. He, "Realization of nano static strain sensing with fiber Bragg gratings interrogated by narrow linewidth tunable lasers," Optics Express, vol. 19, no. 21, pp. 20214-20223, 2011.

[13] K. Yamamura, O. Sano, H. Utada, Y. Takei, S. Nakao, and Y. Fukao, "Long-term observation of in situ seismic velocity and attenuation," Journal of Geophysical Research: Solid Earth, vol. 108, no. B6, pp. 2317, 2003.

[14] Q. Liu, T. Tokunaga, K. Mogi, H. Matsui, H. F. Wang, T. Kato, et al., "Ultra-high resolution multiplexed fiber Bragg grating sensor for crustal strain monitoring," IEEE Photonics Journal, vol. 4, no. 3, pp. 996-1003, 2012.

[15] Q. Liu, T Tokunaga, and Z. He, "Ultra-highresolution large-dynamic-range optical fiber static strain sensor using Pound-Drever-Hall technique," Optics Letters, vol. 36, no. 20, pp. 4044-4046, 2011.

[16] Q. Liu, T Tokunaga, and Z. He, "Sub-nano resolution static strain fiber sensor using a sideband interrogation technique," Optics Letters, vol. 37, no. 3, pp. 434-436, 2012. 